\title{
Dopamine D1 and D2 receptors in the nucleus accumbens core and shell mediate Pavlovian-instrumental transfer
}

\author{
Anja Lex and Wolfgang Hauber ${ }^{1}$ \\ Abteilung Tierphysiologie, Biologisches Institut Universität Stuttgart, D-70550 Stuttgart, Germany
}

\begin{abstract}
Pavlovian stimuli previously paired with food can markedly elevate the rate of food-reinforced instrumental responding. This effect, termed Pavlovian-instrumental transfer (PIT), depends both on general activating and specific cueing properties of Pavlovian stimuli. Recent evidence suggests that the general activating properties of Pavlovian stimuli are mediated by mesoaccumbens dopamine systems; however, the role of NAC dopamine D1 and D2 receptors is still unknown. Here we examined the effects of a selective dopamine D1 and D2 receptor blockade in the shell and core subregion of the NAC on general PIT. Rats were trained to press a single lever for food, and the effect of a single Pavlovian stimulus previously associated with the same food on performance of that lever was measured in extinction. Results reveal that PIT, that is, the increase in instrumental responding during presentation of the Pavlovian stimulus, was reduced by microinjections of the dopamine D1 receptor antagonist $\mathrm{SCH}-23390$ and, less pronounced, by microinjections of the dopamine D2 receptor antagonist raclopride into the NAC core or shell, respectively. Our data suggest that dopamine D1 and D2 receptors in the NAC core and shell mediate the general activating effects of Pavlovian stimuli on instrumental behavior.
\end{abstract}

Pavlovian stimuli predictive of reward can exert a powerful influence on the selection and initiation of instrumental behavior. For instance, presentation of stimuli previously associated with food reward can invigorate instrumental responding, an effect termed Pavlovian-instrumental transfer (PIT) (Estes 1943; Lovibond 1983; Colwill and Rescorla 1988). Pavlovian-instrumental transfer is governed by complex interactions of associative processes that are not completely understood at present (for review, see Dickinson and Balleine 2002; Ostlund and Balleine 2007). One mechanism by which Pavlovian stimuli might influence the vigor of an action is to create a general appetitive arousal that elevates instrumental responding (e.g., Rescorla and Solomon 1967). Furthermore, Pavlovian stimuli can act to selectively potentiate those actions with which they share an outcome, that is, sucrose-predictive stimuli increase responses associated with sucrose reward more than responses that earn different rewards (e.g., Rescorla and Solomon 1967; Trapold and Overmier 1972; Kruse et al. 1983; Corbit et al. 2001, 2007). Thus, Pavlovian stimuli also activate the memory of the sensory-specific properties of the associated outcome and selectively elevate instrumental behavior that leads to the same outcome.

The influence of general activating and specific cueing properties of Pavlovian stimuli can be dissociated by different experimental protocols and may be mediated in part by separate neural circuits. The amygdala is one key structure that has long been implicated in mediating the general and outcome-specific PIT (Blundell et al. 2001; Holland and Gallagher 2003; Corbit and Balleine 2005). Specifically, lesion studies indicated that the central nucleus of the amygdala is involved in controlling the general form of PIT, the basolateral part of the amygdala, in controlling the outcome-specific form (Corbit and Balleine 2005). The nucleus accumbens (NAC) is another key structure that plays a

\footnotetext{
1Corresponding author.
}

E-mail hauber@bio.uni-stuttgart.de; fax 49-711-685-65090.

Article is online at http://www.learnmem.org/cgi/doi/10.1101//m.978708. critical role in PIT (Corbit et al. 2001; Hall et al. 2001; de Borchgrave et al. 2002). Notably, lesion of the core subregion of NAC impaired the general motivational effects of reward-predictive stimuli in PIT, whereas lesion of the shell subregion of the NAC abolished their selective cueing effects (Corbit et al. 2001; Hall et al. 2001). Furthermore, recent studies implicated the ventral tegmental area (VTA) to support both the general and the outcomespecific PIT (Murschall and Hauber 2006; Corbit et al. 2007).

While it is well established that general PIT depends crucially on the integrity of the basolateral amygdala, NAC core, and VTA, little is known yet about its neurochemical basis. Recent studies suggest a role for dopamine (DA) as a systemic blockade of DA receptors (Dickinson et al. 2000) as well as transient inactivation (Murschall and Hauber 2006; Corbit et al. 2007) or DA depletion (El-Amamy and Holland 2007) of the VTA abolished, while stimulation of mesoaccumbens DA neurotransmission increased general PIT (Wyvell and Berridge 2000). These studies point to an important role for mesoaccumbens DA transmission in general PIT; however, it is unknown whether D1 and D2 receptor-mediated signaling in the NAC core, NAC shell, or both is required.

Here we examined the effects of a selective blockade of D1 and D2 receptors in the NAC shell and core on the general form of PIT. Rats were food deprived and trained to press a single lever for food, and the effect of a single Pavlovian stimulus previously associated with the same food on performance of that lever was measured in extinction. Prior to testing, animals received microinjections of the D1 receptor antagonist SCH23390 or of the D2 receptor antagonist raclopride into the NAC core or shell, respectively.

\section{Results}

\section{Histology}

The location of the injection cannulae tips within the NAC core and NAC shell is shown in Figure 1. All animals were included in the behavioral analysis. 

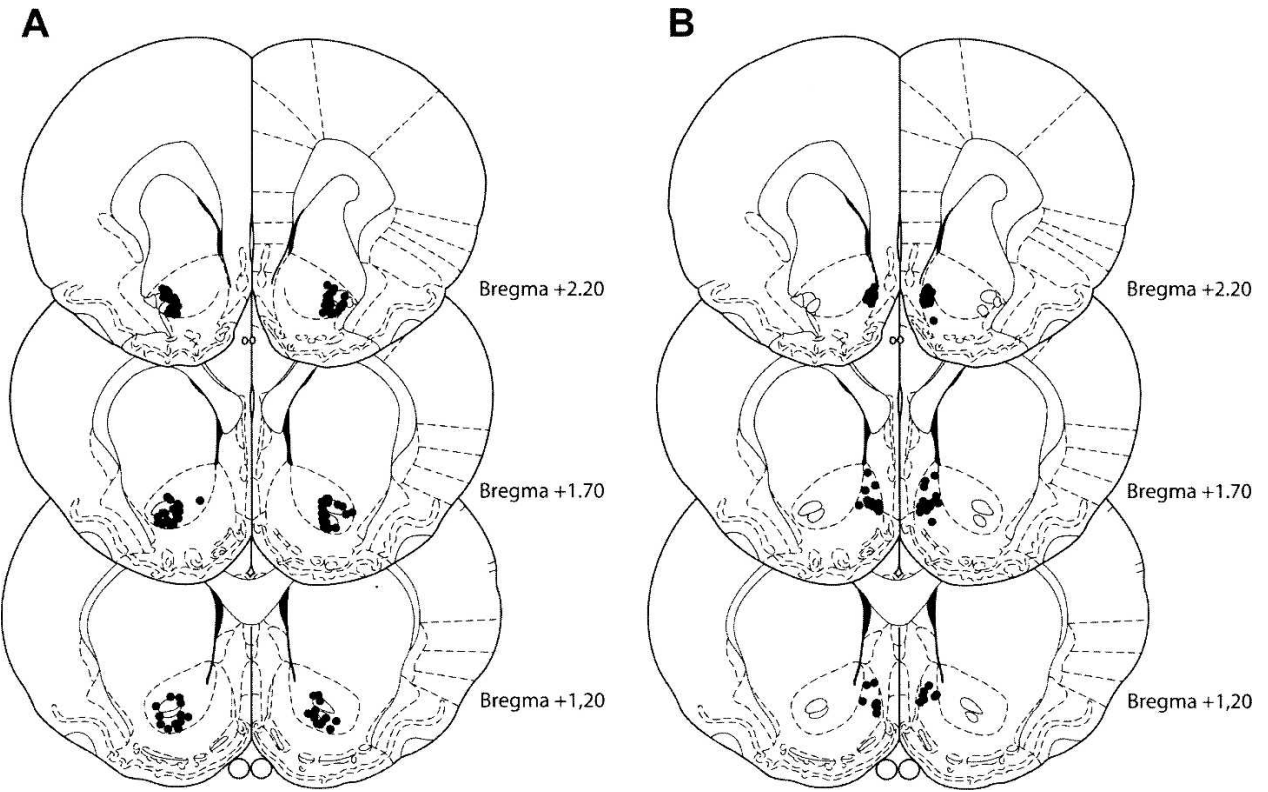

Figure 1. Cannulae placement in the NAC core and shell. The schematics depict the location of the injection cannulae tips $(\bullet)$ in the NAC core $(A)$ and shell (B) for all rats. Plates are adaptations from the atlas of Paxinos and Watson (1997) with permission from Elsevier $\odot 2008$. The number beside each plate corresponds to millimeters anterior to bregma.

\section{Pavlovian training}

Stimulus-directed behavior during Pavlovian training was measured by calculating the approach ratio, that is, the percentage of time spent in the food receptacle during CS+ versus the percentage of time spent in the food receptacle during ISI as follows (e.g., Cardinal et al. 2003): approach ratio $=(\% \mathrm{CS}+) /(\% \mathrm{CS}++\%$ ISI $)$.
During Pavlovian training, the mean approach ratio from all subjects with cannulae placement in the NAC core $(n=57)$ and NAC shell $(n=35)$ significantly increased as shown in Figure 2A; that is, the time spent in the food receptacle during CS+ presentation relative to ISI became increasingly higher. One-way ANOVAs conducted on the training data revealed a significant effect of training day for animals with cannulae placement in the NAC core $\left(F_{(11,616)}=19.12 ; P<0.001\right)$ and in the NAC shell $\left(F_{(11,374)}=8.02\right.$; $P<0.001$ ).
B Instrumental Training

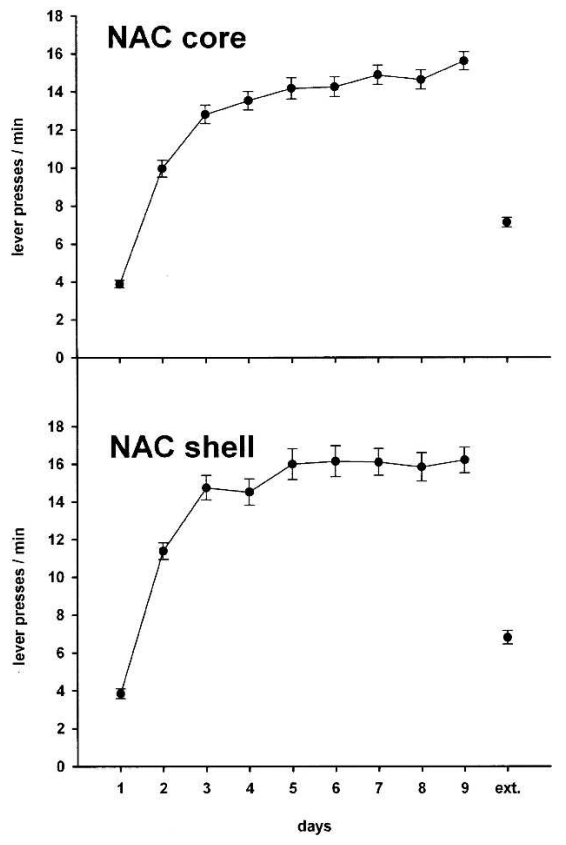

Figure 2. Pavlovian and instrumental training of animals with cannulae placement in the NAC core $(n=57)$ and shell $(n=35)$. (A) Pavlovian training. Mean approach ratio $( \pm$ SEM), that is, the ratio of time spent in the food receptacle during CS+ and time spent in the food receptacle during ISI, across $12 \mathrm{~d}$ of training. (B) Instrumental training. Mean lever presses ( \pm SEM) across $9 \mathrm{~d}$ of training and during a subsequent extinction session without reinforcement (ext).

\section{Instrumental training}

The number of lever presses per minute for all subjects with cannulae placement in the NAC core and NAC shell across days of training is displayed in Figure 2B. All animals acquired instrumental responding over $9 \mathrm{~d}$. One-way ANOVAs conducted on the training data revealed a significant effect of training day for animals with cannulae placement in the NAC core $\left(F_{(8,904)}=331.22 ; P<0.001\right)$ and in the NAC shell $\left(F_{(8,376)}=146.03\right.$; $P<0.001)$. Responding in the NAC core/ shell group increased from $3.88 \pm 0.20$ / $3.84 \pm 0.26$ lever presses per minute on day 1 to $15.66 \pm 0.48 / 16.20 \pm 0.68$ on day 9 , and in the subsequent extinction session instrumental responses decreased to $7.17 \pm 0.26 / 6.81 \pm 0.36$ lever presses/min.

\section{Transfer test: D1 and D2 receptor blockade in the NAC core}

\section{Infusion of SCH-23390}

In rats with intra-NAC core infusion of saline, the CS+ enhanced lever pressing 
relative to the nonreinforced stimulus (CSo) and ISI, while in rats with intra-NAC core infusion of SCH-23390, this transfer effect was abolished and the baseline rate of lever responding was reduced (Fig. 3A).

A three-way ANOVA with treatment (saline, $0.5 \mu \mathrm{g}$ of SCH23390 and $0.75 \mu \mathrm{g}$ of SCH-23390) and CS identity (tone and white noise) as between-subject factors and stimulus (ISI, CSo, and $\left.\mathrm{CS}_{+}\right)$as within-subjects factor revealed a main effect of treatment $\left(F_{(2,29)}=10.39 ; P<0.001\right)$, stimulus $\left(F_{(2,58)}=14.09\right.$; $P<0.001$ ), a stimulus $\times$ CS identity interaction (greater enhancement with the white noise; $\left.F_{(2,58)}=6.23 ; P<0.01\right)$ and stimulus $\times$ treatment interaction $\left(F_{(4,58)}=4.27 ; P<0.01\right)$. Simple effects analyses revealed a significant effect of stimulus type in animals treated with saline $\left(F_{(2,24)}=22.23 ; P<0.001\right)$, and subsequent post hoc comparisons yielded higher responding during CS+ relative to ISI and CSo (ISI: $P<0.001$; CSo: $P<0.001$ ). In contrast, no effect of stimulus type was observed with $0.5 \mu \mathrm{g}$ of SCH-23390 $\left(F_{(2,20)}=3.01 ; P=0.072\right)$ and $0.75 \mu \mathrm{g}$ of SCH-23390

\section{A}

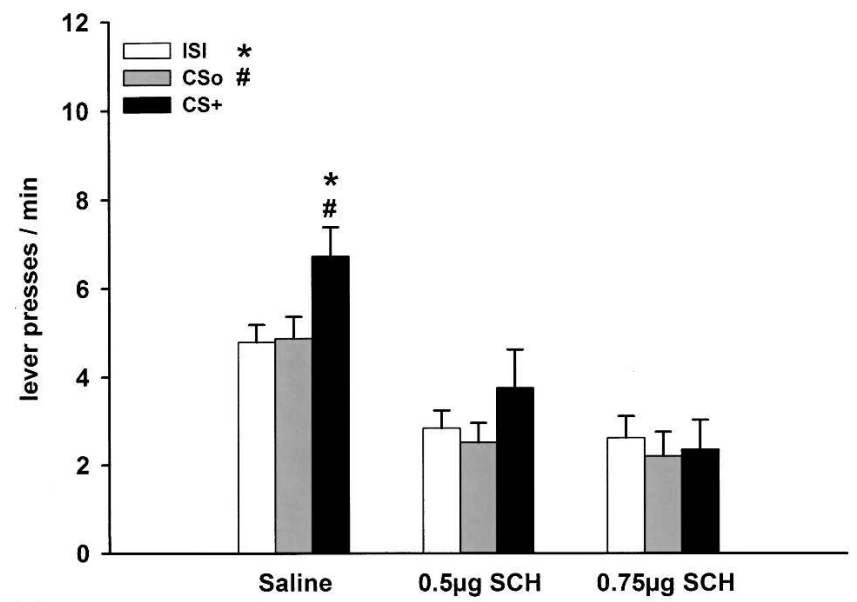

B

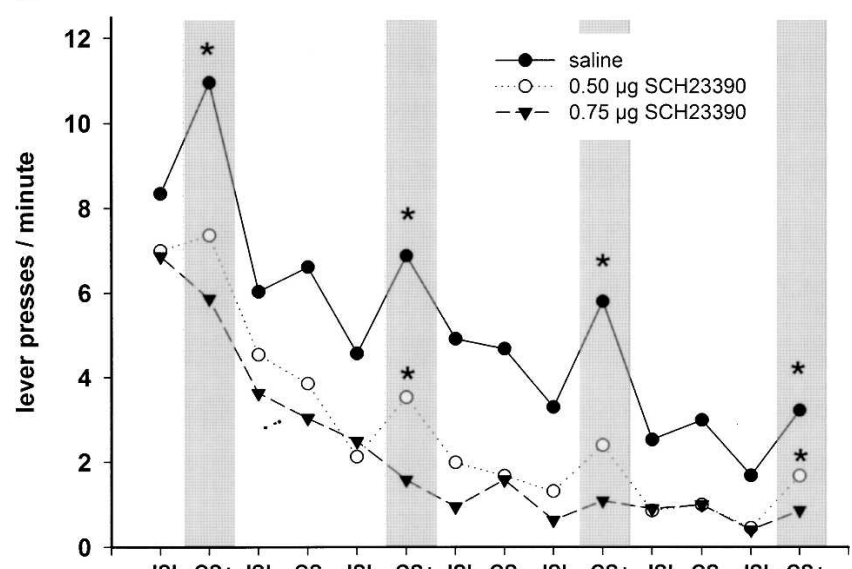

ISI CS+ ISI CS- ISI CS+ ISI CS- ISI CS+ ISI CS- ISI CS+

Figure 3. Pavlovian-instrumental transfer following infusion of D1 receptor antagonists into the NAC core. $(A)$ Effect of intra-NAC core infusion of saline $(n=13), 0.5 \mu \mathrm{g}$ of SCH-23390 $(n=11)$, and $0.75 \mu \mathrm{g} \mathrm{SCH}$ $23390(n=11)$ on mean lever presses $( \pm$ SEM) during presentation of $\mathrm{CS}+, \mathrm{CS}$, and ISI. The CS+ significantly elevated responding relative to CSo and ISI after saline $(P<0.001)$, but not after $0.5 \mu \mathrm{g}$ and $0.75 \mu \mathrm{g}$ of SCH-23390 ( $P>0.05$, ANOVA followed by a post hoc Tukey HSD test). $(B)$ Mean lever presses as a function of the temporal order of stimulus presentation during the test session. $\left(^{*}\right) P<0.05 C S+$ versus preceding ISI (planned contrast analysis). (CS+) Conditioned stimulus; (CSo) neutral stimulus; (ISI) intertrial interval (2 min in duration, respectively).
$\left(F_{(2,20)}=0.57 ; P=0.576\right)$, indicating that both doses of SCH-23390 completely abolished the transfer effect. Furthermore, simple effect analyses revealed an effect of treatment during ISI $\left(F_{(2,32)}=8.31\right.$; $P<0.01)$, CSo $\left(F_{(2,32)}=9.01 ; P<0.001\right)$, and CS $+\left(F_{(2,32)}=9.76\right.$; $P<0.001)$, indicating that baseline responding during ISI and CSo was reduced by drug application.

An inspection of the time course of stimulus effects showed that in saline-treated animals, presentation of the CS+ increased instrumental behavior relative to the preceding ISI throughout the test session. In contrast, in animals that received the high dose of SCH23390, the transfer effect was completely abolished, whereas in animals that received the low dose of SCH23390, there was a partial reduction of the transfer effect (Fig. 3B).

\section{Infusion of raclopride}

In rats with intra-NAC core infusion of saline, the CS+ enhanced lever pressing relative to CSo and ISI, while in rats with intraNAC core infusion of raclopride, this transfer effect was impaired, and the baseline rate of lever responding was reduced (Fig. 4A).

An ANOVA with treatment (saline, $0.5 \mu \mathrm{g}$ of raclopride, and $1.0 \mu \mathrm{g}$ of raclopride), CS identity (tone and white noise) as betweensubject factors and stimulus (ISI, CSo, and CS+) as within-subject factor revealed an effect of treatment $\left(F_{(2,29)}=3.64 ; P<0.05\right)$, stimulus $\left(F_{(2,58)}=23.44 ; P<0.001\right)$, and stimulus $\times$ CS identity interaction (greater enhancement with the white noise; $F_{(2,58)}=8.05$; $P<0.001)$. Simple effects analyses revealed a significant effect of stimulus type in saline $\left(F_{(2,24)}=22.23 ; P<0.001\right)$ and $0.5 \mu \mathrm{g}$ of raclopride $\left(F_{(2,20)}=4.87 ; P<0.05\right)$ treated animals. Subsequent post hoc comparisons indicated increased responding during CS+ relative to ISI and CSo (ISI: $P<0.001$; CSo: $P<0.001$ ) in saline-treated animals, increased responding during CS+ relative to ISI $(P<0.05)$ but not to CSo $(P=0.073)$ in $0.5 \mu \mathrm{g}$ of raclopridetreated animals, thus indicating a reduced transfer effect in this latter group. No effect of stimulus type, and hence no transfer effect, could be observed in the $1.0 \mu \mathrm{g}$ of raclopride-treated animals $\left(F_{(2,20)}=3.02 ; P=0.072\right)$. In addition, simple effects analyses revealed that raclopride caused a significant decrease of responding during ISI $\left(F_{(2,32)}=6.89 ; P<0.01\right)$, but not during CSo $\left(F_{(2,32)}=2.87 ; P=0.071\right)$ or $\mathrm{CS}+\left(F_{(2,32)}=1.77 ; P=0.187\right)$.

In animals that received the low dose of raclopride, significant transfer effects were observed throughout the test session. In animals treated with the high dose of raclopride, transfer effects were also seen; however, they were only in part statistically significant (Fig. 4B).

\section{Transfer test: D1 and D2 receptor blockade in the NAC shell}

Infusion of SCH-23390

In rats with intra-NAC shell infusion of saline, lever pressing during CS+ was enhanced relative to the CSo and ISI, while infusion of low and high doses of SCH-23390 impaired this transfer effect (Fig. 5A).

The overall ANOVA with treatment (saline, $0.5 \mu \mathrm{g}$ of $\mathrm{SCH}-$ 23390 and $0.75 \mu \mathrm{g}$ of SCH-23390), CS identity (tone and white noise), and stimulus (ISI, CSo, and CS+) revealed an effect of treatment $\left(F_{(2,15)}=4.89 ; P<0.05\right)$, stimulus $\left(F_{(2,30)}=10.36\right.$; $P<0.001$ ), stimulus $\times$ CS identity interaction (greater enhancement with the white noise; $\left.F_{(2,30)}=4.72 ; P<0.05\right)$, and a stimulus $\times$ treatment interaction $\left(F_{(4,30)}=3.95 ; P<0.05\right)$. Subsequent analyses of the simple effects revealed a significant effect of stimulus type in the saline-treated group $\left(F_{(2,14)}=16.86\right.$; $P<0.001$ ), and post hoc comparisons yielded higher responding during CS+ relative to ISI and CSo (ISI: $P<0.001$; CSo: $P<0.001$ ). In contrast, no effect of stimulus type was observed with $0.5 \mu \mathrm{g}$ of SCH-23390 $\left(F_{(2,12)}=1.22 ; P=0.330\right)$ and $0.75 \mu \mathrm{g}$ of SCH$23390\left(F_{(2,10)}=0.14 ; P=0.871\right)$, indicating that both doses of 
A

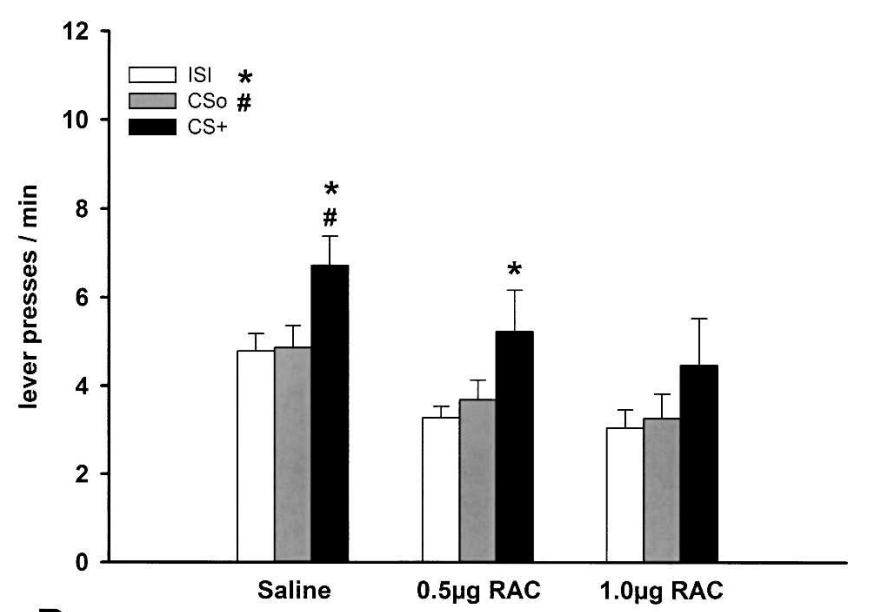

B

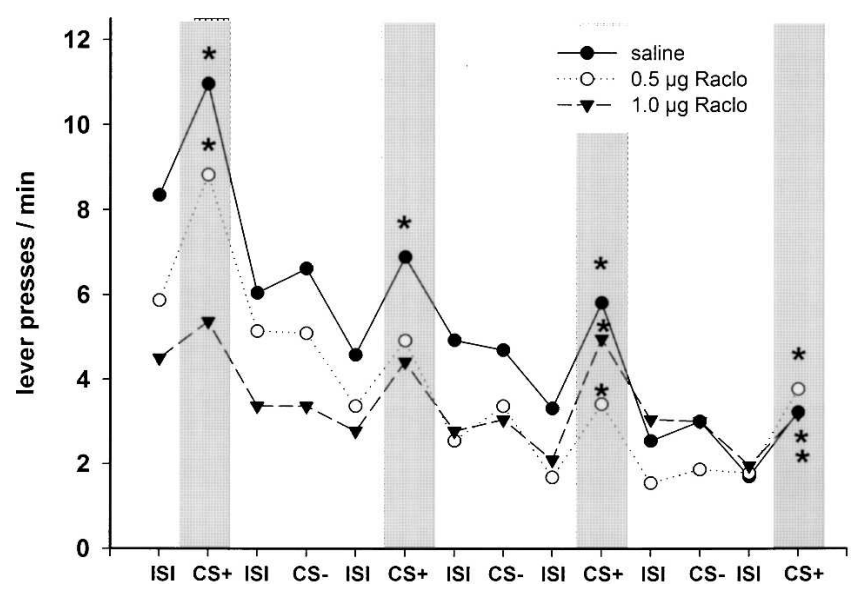

Figure 4. Pavlovian-instrumental transfer following infusion of D2 receptor antagonists into the NAC core. $(A)$ Effect of intra-NAC core infusion of saline $(n=13), 0.5 \mu \mathrm{g}$ of raclopride $(n=11)$, and $1.0 \mu \mathrm{g}$ of raclopride $(n=11)$ on mean lever presses ( \pm SEM) during presentation of $\mathrm{CS}+, \mathrm{CSO}$, and ISI. The CS+ significantly elevated responding relative to CSo and ISI after saline infusion $(P<0.001)$. After $0.5 \mu \mathrm{g}$ of raclopride injection responding during $C S+$ was significantly increased relative to ISI $(P<0.05)$ but not to CSo. Following infusion of $1.0 \mu \mathrm{g}$ of raclopride, responding during $\mathrm{CS}+$ was not increased relative to CSo and ISI $(P>0.05$, ANOVA followed by a post hoc Tukey HSD test). (\#) $P<0.05$ CS+ versus CSo; $\left(^{*}\right) P<0.05$ CS+ versus ISI. (B) Mean lever presses as a function of the temporal order of stimulus presentation during the test session. ( $\left.{ }^{*}\right) P<0.05 C S+$ versus preceding ISI (planned contrast analysis). (CS+) Conditioned stimulus; (CSo) neutral stimulus; (ISI) intertrial interval (2 min in duration, respectively).

SCH-23390 abolished the transfer effect. Furthermore, analyses of the simple effects revealed no effect of treatment on responding during ISI $\left(F_{(2,28)}=3.07 ; P=0.071\right)$ and CSo $\left(F_{(2,28)}=1.86\right.$; $P=0.185)$ but during CS $+\left(F_{(2,18)}=7.071 ; P<0.01\right)$, indicating that SCH-23390 did not significantly affect baseline responding.

An inspection of the time course of stimulus effects showed that in saline-treated animals, presentation of the CS+ increased instrumental behavior throughout the test session. In contrast, in animals that received the high dose of SCH23390, transfer effects were completely abolished throughout the session, whereas in animals that received the low dose of SCH23390, transfer effects can be seen, which, however, reached no statistical significance (Fig. 5B).

\section{Infusion of raclopride}

In rats subjected to intra-NAC shell infusion of saline, lever pressing was enhanced during CS+ relative to CSo and ISI, while this transfer effect was impaired in rats treated with low and high doses of raclopride (Fig. 6B).

An ANOVA with treatment (saline, $0.5 \mu \mathrm{g}$ of raclopride, and $1.0 \mu \mathrm{g}$ of raclopride) and CS identity (tone and white noise) and stimulus (ISI, CSo, and CS+) revealed an effect of stimulus $\left(F_{(2,32)}=16.17 ; P<0.001\right)$ and a stimulus $\times$ treatment interaction $\left(F_{(4,32)}=3.16 ; P<0.05\right)$. Simple effects analyses revealed a significant effect of stimulus type during application of saline $\left(F_{(2,14)}=16.86 ; P<0.001\right)$, and post hoc comparisons indicated that responding during CS+ was increased relative to ISI $(P<0.001)$ and CSo $(P<0.001)$. In contrast, no effect of stimulus type was observed with $0.5 \mu \mathrm{g}$ of raclopride $\left(F_{(2,12)}=1.38\right.$; $P=0.288$ ). Surprisingly, simple effects analyses revealed a significant effect of stimulus type after infusion of $1.0 \mu \mathrm{g}$ of raclopride

\section{A}

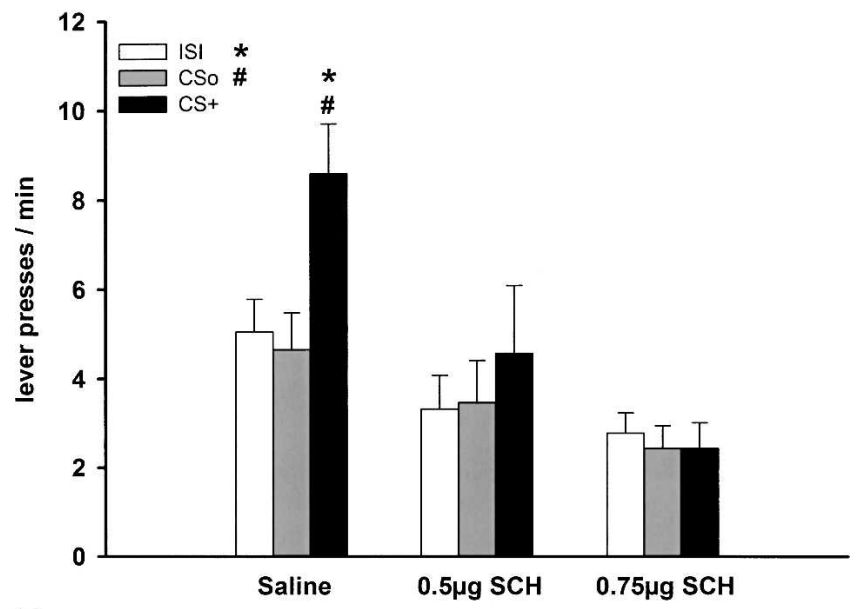

B

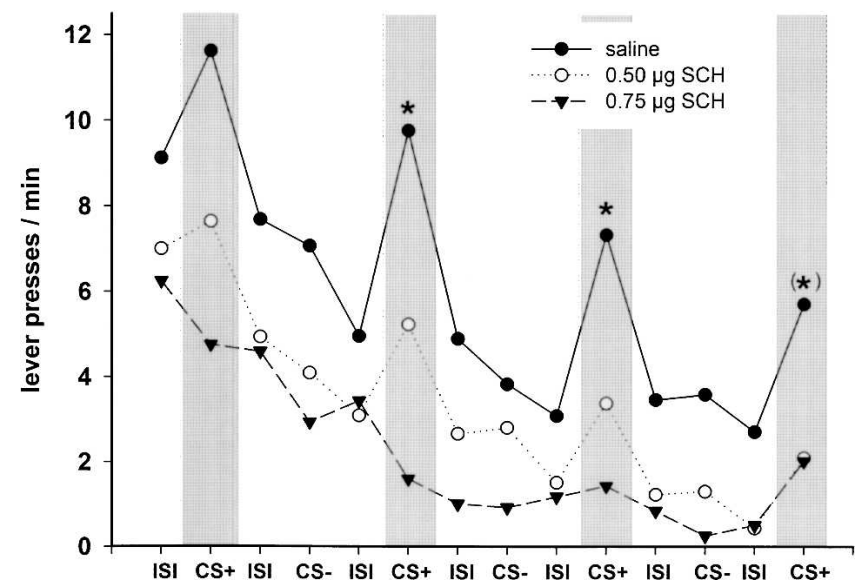

Figure 5. Pavlovian-instrumental transfer following infusion of D1 receptor antagonists into the NAC shell. (A) Effect of intra-NAC shell infusion of saline $(n=8), 0.5 \mu \mathrm{g}$ of $\mathrm{SCH}-23390(n=7)$ and $0.75 \mu \mathrm{g}$ of SCH$23390(n=6)$ on mean lever presses ( \pm SEM) during presentation of CS+, CSo, and ISI. The CS+ significantly elevated responding relative to CSo and ISI after saline $(P<0.001)$, but not after $0.5 \mu \mathrm{g}$ and $0.75 \mu \mathrm{g}$ of SCH-23390 $(P>0.05$, ANOVA followed by a post hoc Tukey HSD test) (B) Mean lever presses as a function of the temporal order of stimulus presentation during the test session. ${ }^{*} P<0.05 ;\left({ }^{*}\right) P=0.57 \mathrm{CS}+$ versus preceding ISI (planned contrast analysis). (CS+) Conditioned stimulus; (CSo) neutral stimulus; (ISI) intertrial interval (2 min in duration, respectively). 
A

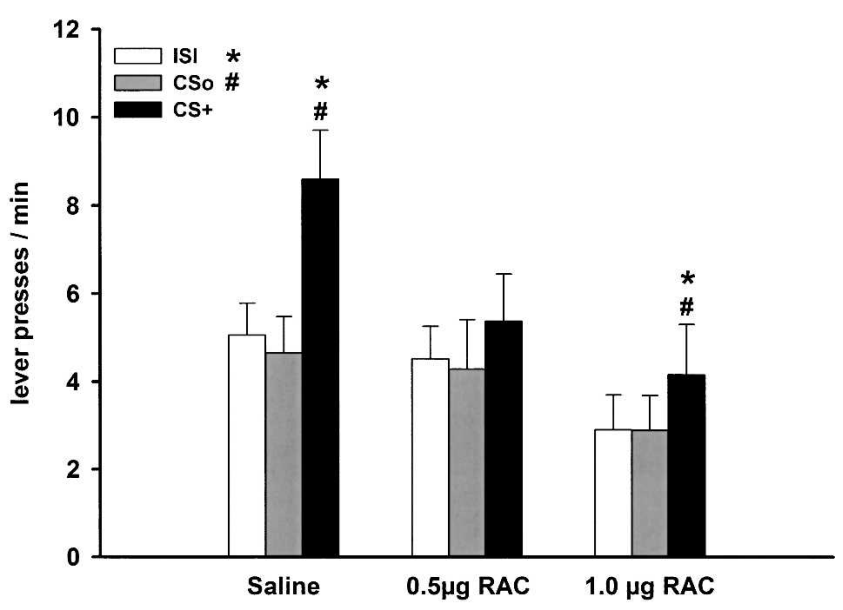

B

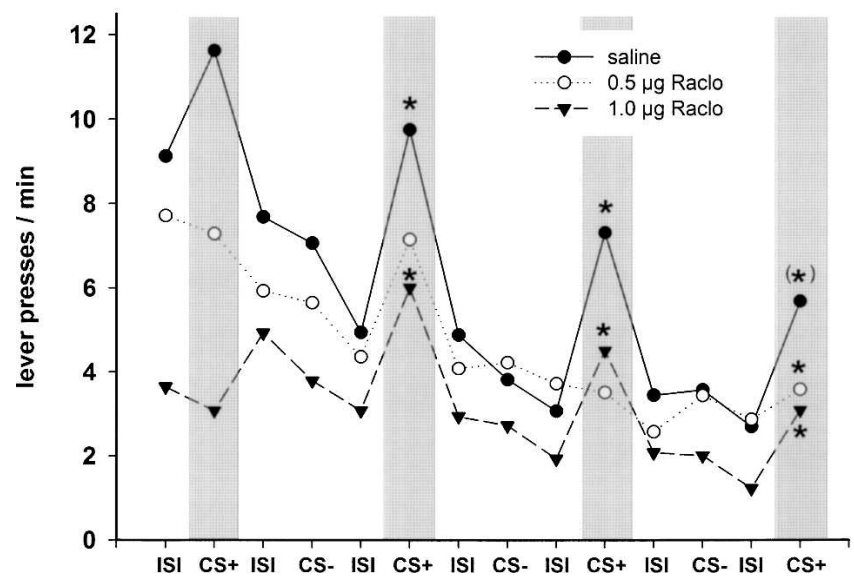

Figure 6. Pavlovian-instrumental transfer following infusion of D2 receptor antagonists into the NAC shell. $(A)$ Effect of intra-NAC shell infusion of saline $(n=8), 0.5 \mu \mathrm{g}$ of raclopride $(n=7)$, and $1.0 \mu \mathrm{g}$ of raclopride $(n=7)$ on mean lever presses $( \pm$ SEM) during presentation of CS+, CSo, and ISI. The CS+ significantly elevated responding relative to CSO and ISI after saline $(P<0.001)$ and $1.0 \mu \mathrm{g}$ of raclopride $(P<0.05)$, but not after $0.5 \mu \mathrm{g}$ of raclopride $(P>0.05$, ANOVA followed by a post hoc Tukey HSD test). (B) Mean lever presses as a function of the temporal order of stimulus presentation during the test session. ${ }^{*} P<0.05 ;\left(^{*}\right) P=0.057 \mathrm{CS}+$ versus preceding ISI (planned contrast analysis). (CS+) Conditioned stimulus; (CSo) neutral stimulus; (ISI) intertrial interval (2 min in duration, respectively).

$\left(F_{(2,12)}=5.10 ; P<0.05\right)$, and post hoc comparisons indicated that responding during $\mathrm{CS}+$ was increased relative to ISI $(P<0.05)$ and CSo $(P<0.05)$. This significant effect was largely due to one outlier in the treatment group that, for unknown reasons, had a pronounced transfer effect $(+4.06$ lever presses/min relative to CSo) not observed in any other animal that received the high dose of raclopride $(n=6$; transfer effect of -0.50 to +1.94 lever presses/min relative to CSo). Further analyses of the simple effects revealed an effect of raclopride on responding during CS+ $\left(F_{(2,19)}=4.35 ; P<0.05\right)$ presentation, but not during ISI $\left(F_{(2,19)}=2.19 ; P=0.139\right)$ and CSo $\left(F_{(2,19)}=1.01 ; P=0.381\right)$, indicating that baseline responding was not significantly affected.

An analysis of the time course of CS+ effects revealed that in animals that received the high dose of raclopride, significant transfer effects were observed during the test session. In animals treated with the low dose of raclopride, significant trans- fer effects were only observed during one out of four CS+ presentations (Fig. 6B).

\section{Discussion}

The present study demonstrates that a selective D1 blockade and, less pronounced, a D2 receptor blockade both in the NAC core and shell reduced general PIT. These findings provide support to the notion that D1 and D2 receptor-mediated signaling in two major subregions of the NAC mediates the excitatory impact of appetitive Pavlovian stimuli on instrumental behavior.

The possibility that drug diffusion into adjacent subregions contributes to drug effects on PIT cannot be discounted. However, quantitative autoradiography revealed a low rate of diffusion of SCH23390 from the site of an intracerebral injection suggesting a local action, at least in the first 30 min after microinjection (Caine et al. 1995). Furthermore, intra-NAC core and shell infusions of SCH23390 and raclopride at even higher doses and volumes as used here produced dissociable behavioral effects (Baldo et al. 2002; Anderson et al. 2003; Bari and Pierce 2005), indicating that the spread of drugs was relatively limited. Thus, SCH23390- and raclopride-induced effects on PIT observed here may reflect drug actions largely limited to the NAC core and shell, respectively.

Drug infusion into the NAC core significantly reduced lever press rates during ISI, while drug infusion into the NAC shell had less pronounced effects. Reduced lever press rates could indicate an impaired response capacity that accounts for attenuated PIT. Yet, as measured in extinction, lever press rates in PIT are generally low and may thus not challenge response capacity excessively. Furthermore, in animals that received intra-NAC core infusions, the absolute rates of lever presses during ISI were not abnormally low but correspond to those observed in animals that received intra-NAC shell infusions or to those reported in previous studies in intact and treated animals (e.g., Murschall and Hauber 2006; El-Amamy and Holland 2007). Most importantly, an inspection of the time course of CS+ effects in animals with drug infusions showed that PIT can be absent already during early CS+ presentations when basal responses rates were high. In contrast, significant PIT was observed during late CS+ presentations when basal response rates were lower. Thus, it appears that PIT is a robust phenomenon that can be elicited even if basal response rates are relatively low. Disruption of PIT, therefore, may not result from a floor effect caused by SCH23390- or racloprideinduced nonspecific impairments of instrumental responding.

\section{NAC core and Pavlovian-instrumental transfer}

In this study, we used a protocol adopted from previous studies (e.g., Hall et al. 2001) that generates a general form of PIT as rats were trained on a single lever, and the effect of a single Pavlovian stimulus on performance of that lever was measured. Instrumental training, in particular under interval schedules of reinforcement as used here, can generate habitual responding (Dickinson et al. 1983). Yet, in the instrumental extinction session subsequent to instrumental training, we observed a significant decrease of lever press rates, indicating that animals were still sensitive to the outcome, that is, did not respond in a habitual manner. In our previous studies using the same protocol (Murschall and Hauber 2005, 2006), lever press rates during instrumental training and transfer testing were moderately lower. Rat sample differences might account for this variability. Furthermore, unlike in our previous studies (Murschall and Hauber 2005, 2006), the white noise $\mathrm{CS}+$ was somewhat more effective than the tone CS+. However, the tone CS+ per se always produced reliable PIT; therefore, CS+ identity effects might be largely neglected.

Intra-NAC core infusion of SCH23390 abolished PIT, sug- 
gesting a critical role of D1 receptor-mediated signaling in the NAC core to mediate the general excitatory effects of a Pavlovian stimulus on instrumental responding. Intra-NAc core blockade of D2 receptors also abolished PIT during early CS+ presentations, but, relative to SCH23390, the effects of raclopride were less pronounced. This finding tentatively points to a less prominent role of D2 receptor in the NAC core in controlling the general PIT. Further dose-response studies may be warranted to address the relative contribution of D1 and D2 receptors in PIT. Yet, our pilot experiments indicate that markedly higher doses of intra-NAC raclopride or SCH23390 as used here produce pronounced effects on response initiation.

Our findings are in accordance with lesion studies that have implicated the NAC core in the general form of PIT (Hall et al. 2001) and add to recent data suggesting a role for DA in PIT. For instance, systemic blockade of DA receptors (Dickinson et al. 2000) as well as transient inactivation (Murschall and Hauber 2006; Corbit et al. 2007) or DA depletion (El-Amamy and Holland 2007) of the VTA abolished, while stimulation of mesoaccumbens DA neurotransmission increased general PIT (Wyvell and Berridge 2000). Furthermore, a large body of evidence has implicated DA in the NAC core in processing of food CS in a variety of other paradigms that involve actions of Pavlovian stimuli (Di Chiara and Bassareo 2007; Nicola 2007). For instance, microdialysis studies demonstrated that presentation of an appetitive food CS increased DA transmission in the NAC core (Bassareo and Di Chiara 1997; Cheng et al. 2003). Thus, a CS-induced DA release acting on NAC core D1 and D2 receptors may represent a neurochemical substrate of the invigorating effects of an appetitive CS on instrumental behavior observed here. In line with this account, D1 and D2 receptor activity in the NAC core regulates the rate (Yun et al. 2004) and initiation (Calaminus and Hauber 2007) of instrumental responses to appetitive discriminative cues that may serve in part as Pavlovian stimuli (Colwill and Rescorla 1988). Our findings are also consistent with a proposed neural circuitry for general PIT involving NAC DA release that is regulated by projections from the central nucleus of the amygdala (CeA) to midbrain DA neurons (Parkinson et al. 2000; Hall et al. 2001; Cardinal et al. 2002). However, this circuit model may be too simplistic as recent studies provide evidence for additional structures to be involved in the regulation of general PIT. For instance, in rats there is little evidence for strong monosynaptic projections from the CeA to the VTA (Zahm et al. 1999); thus, polysynaptic pathways linking the CeA and the VTA, for example, through the lateral hypothalamus or frontal cortex (Zahm 2006), may regulate PIT as well. In addition, the finding that lesions that disconnected the CeA from the VTA facilitated PIT relative to unilateral VTA lesions (El-Amamy and Holland 2007) is at variance with the notion that PIT requires direct interactions between both structures. Furthermore, El-Amamy and Holland (2007) showed that lesions of the substantia nigra pars compacta had debilitating effects on PIT, indicating that the nigral DA innervation of the NAC or other target areas of nigrostriatal DA fibers such as the dorsal striatum may be important to PIT. In line with this notion, Corbit and Janak (2007) recently reported that the dorsolateral striatum seems to play a prominent role in allowing excitatory stimuli to invigorate response output. At present, it is not clear how this observation can be reconciled with the CeA-VTA-NAC circuit model of general PIT. However, this finding points to the possibility that the DA innervation of other structures involved in stimulus-guided responding, for example, the dorsal striatum, is critical for PIT.

\section{NAC shell and Pavlovian-instrumental transfer}

Our data further show that PIT was largely absent in animals that received intra-NAC shell infusions of SCH23390. Thus D1 recep- tors in the NAC shell may play a critical role in mediating general PIT. Results obtained with intra-NAc shell infusion of raclopride were less clear-cut. The low dose of raclopride abolished PIT during most CS+ presentations, while the high dose was effective only during the initial CS+ presentation. These findings indicate a complex dose-response profile of NAC shell D2 receptor activity in PIT. As already pointed out, studies using other doses of raclopride may be warranted, yet our preliminary findings suggest that higher doses of intra-NAC raclopride markedly interfere with response initiation.

In line with our observations, stimulation of DA transmission in the NAC shell enhanced general PIT (Wyvell and Berridge 2000; Pecina et al. 2006). Notably, using the same PIT protocol as in this study, Hall et al. (2001) revealed that cell body lesions of the NAC shell had only moderate effects. Although reduced by NAC shell lesions, general PIT showed a trend toward significance (Hall et al. 2001), while in the present study, the debilitating effects of NAC shell manipulations on general PIT were, for unknown reasons, more pronounced. A large number of studies showed that presentation of drug-predictive CS stimulate DA efflux in the NAC shell (Di Chiara and Bassareo 2007; but see Ito et al. 2000), whereas food-predictive CS had more variable effects. Cheng et al. (2003) and Datla et al. (2002) demonstrated that a CS signaling food pellets increased DA efflux both in the NAC shell and core, while Bassareo et al. (2002) observed an enhanced DA transmission in the NAC core only. However, unlike Bassareo et al. (2002), Cheng et al. (2003) used a restricted feeding regimen that can facilitate DA release to food-predictive CS (Wilson et al. 1995). In view of these findings, the experimental protocol used here is likely to stimulate DA transmission in the NAC shell as our animals were maintained on a restricted feeding regimen. Furthermore, our PIT procedure used a discrete unimodal CS predictive of food pellets in a Skinner box, a protocol shown to readily facilitate DA release in the NAC shell (Cheng et al. 2003). Recent data further indicate a role for the NAC shell in mediating the motivational effects of food CS on behavior. Pecina et al. (2006) revealed that corticotrophin-releasing factor (CRF) infusion into the NAC shell enhanced PIT, suggesting that CRF systems could magnify the appetitive motivational arousal associated with CS. Thus, other neurochemical signals than DA converging on the NAC shell could serve to enhance the appetitive motivational arousal of CS, for example, either directly or, possibly, via modulation of D1 and D2 receptor-mediated signaling. Finally, it is important to note that PIT depends both on general activating and specific cueing properties of Pavlovian stimuli. In a general PIT procedure as used here, the Pavlovian stimulus was associated with the same outcome as the instrumental response. Thus, we cannot rule out that a certain amount of outcomespecific transfer was induced. The NAC shell plays an important role in mediating the outcome-specific PIT (Corbit et al. 2001); therefore, the reduced PIT after NAC shell DA antagonism seen here could not only be due to a reduced general excitatory arousal, but to some extent to an impaired retrieval of the specific sensory properties of the outcome as well. In future studies, it will be important to try to separate possible general and outcomespecific transfer effects mediated by the NAC shell DA, for example, by using paradigms that allow for a simultaneous examination of selective and general transfer mechanisms (e.g., Corbit and Balleine 2005).

To summarize, the available evidence suggests that the CeA (Holland and Gallagher 2003; Corbit and Balleine 2005), VTA (Murschall and Hauber 2006; Corbit, et al. 2007), NAC core/shell, and mesoaccumbens DA systems (Wyvell and Berridge 2000; Hall et al. 2001) are major components of a neural circuitry mediating the general form of PIT. However, their interactions are more complex than previously suggested by the simple serial CeA-VTA-NAC 
circuit model and may involve additional structures such as the dorsolateral striatum (Corbit and Janak 2007) and substantia nigra pars compacta (El-Amamy and Holland 2007). Likewise, the neural circuit mediating the outcome-specific PIT appears to be more complex as it not only includes the basolateral amygdala (Blundell et al. 2001; Corbit and Balleine 2005) and NAC shell (Corbit et al. 2001), but, as recently shown, also involves additional structures such as VTA (Corbit et al. 2007) and dorsomedial striatum (Corbit and Janak 2007). Therefore, rather than being strictly segregated, the neural substrates mediating the general and outcome-specific PIT seem to be partially overlapping as, for instance, DA neurons originating in the VTA subserve both forms of PIT (Murschall and Hauber 2006; Corbit et al. 2007; El-Amamy and Holland 2007).

\section{Conclusions}

Taken together, our results suggest that DA, by acting on D1 and D2 receptors both in the shell and core subregion of the NAC, mediates the general motivational impact of Pavlovian stimuli on instrumental responding. These findings provide further support to the notion that one important mechanism through which the NAC influences instrumental behavior is to allow Pavlovian stimuli to energize or activate responding (Cardinal et al. 2002). In addition, they are consistent with the view that mesoaccumbens dopamine activation enhances the conditioned incentive impact of reward cues (Dickinson et al. 2000; Wyvell and Berridge 2000) and demonstrate for the first time that D1 and D2 receptors in the core and shell subregion of the NAC mediate the invigorating effects of Pavlovian stimuli predictive of natural reward.

\section{Materials and Methods}

\section{Subjects}

Subjects were 92 male Lister hooded rats (Harlan Winkelmann) weighing 220-290 $\mathrm{g}$ at the time of surgery. Rats were housed in groups of five animals at $20^{\circ} \pm 2^{\circ} \mathrm{C}$ on a $12: 12$-h light-dark cycle (lights on at 7:00 a.m.) with ad libitum access to water and standard laboratory chow (Altromin). Prior to the onset of behavioral training, food was restricted to $15 \mathrm{~g}$ per animal and day to maintain $\sim 85 \%$ of the animals' free-feeding weight. All animal experiments were conducted according to the German Law on Animal Protection and approved by the proper authorities.

\section{Surgery}

For stereotaxic surgery, animals were anaesthetized with ketamine hydrochloride (120 mg/kg; Bela-Pharm) and xylazine hydrochloride (4 mg/kg Rompun; Bayer) and secured in a Kopf stereotaxic apparatus (Kopf Instruments). Bilateral 14-mm stainless steel guide cannulae $(0.7 \mathrm{~mm}$ outer diameter; Braun Melsung) aimed at either the nucleus accumbens core (NAC core) or shell (NAC shell) were implanted using standard stereotaxic procedures. The coordinates with reference to the atlas of Pellegrino et al. (1981) were: 3.2 anterior bregma, \pm 1.9 lateral to midline and 7.1 ventral from the dura for the NAC core $(n=57)$ and 3.1 anterior bregma, \pm 0.9 lateral to midline and 7.1 ventral from the skull for the NAC shell $(n=35)$ with the toothbar set at $+5.0 \mathrm{~mm}$ above the interaural line. The guide cannulae were occluded by 14-mm stainless steel stylets $(0.4 \mathrm{~mm}$ outer diameter; Braun). Each rat was given at least $5 \mathrm{~d}$ to recover from surgery before the onset of behavioral training.

\section{Drug injection}

Animals received intra-NAC core or shell microinjections of either the selective D1 dopamine receptor antagonist $\mathrm{R}(+)-7$-chloro8-hydroxy-3-methyl-1-phenyl-2,3,4,5-tetrahydro-1H-3benzazepine hydrochloride [R(+)-SCH-23390 hydrochloride; Sigma-Aldrich] or the selective dopamine D2 receptor antagonist 3,5-dichloro- $N$-(1-ethylpyrrolidin-2-ylmethyl)-2-hydroxy-6- methoxybenzamide tartrate salt $[\mathrm{S}(-)$-raclopride (+)-tartrate salt; Sigma-Aldrich]. Drugs were dissolved in physiological saline $(0.9 \%)$ and injected at doses of $0.5 \mu \mathrm{g}$ of SCH-23390, $0.75 \mu \mathrm{g}$ of $\mathrm{SCH}-23390,0.5 \mu \mathrm{g}$ of raclopride, and $1.0 \mu \mathrm{g}$ of raclopride in 0.3 $\mu \mathrm{L}$, respectively; control subjects received microinjections of 0.3 $\mu \mathrm{L}$ of saline. The drug doses were based on previous studies in the literature (e.g., Yun et al. 2004; Calaminus and Hauber 2006) and on pilot studies.

For microinjections, stainless steel stylets were removed, and bilateral injection cannulae $(0.4 \mathrm{~mm}$ outer diameter; Braun) were lowered at the final site of infusion and attached via polyvinylchloride tubing to microliter syringes controlled by a syringe pump (Med Associates). Injection cannulae (length: $18 \mathrm{~mm}$ ) protruded $4 \mathrm{~mm}$ beyond the guide cannulae. All injections were delivered bilaterally over a 1-min interval, and injection cannulae were left in position for a further minute to allow for diffusion. After microinjection, each animal remained in its home cage for an additional 10 min before being placed in the test chamber.

To adapt animals to the microinjection procedure, they received four sham injections including handling, insertion of injection cannulae dummies, and operation of the injection pump (without running an injection) prior to behavioral training on days $3,5,7$, and 9 of instrumental training. On the day before the transfer test, that is, the instrumental extinction session, all animals received a single injection of vehicle $(0.3 \mu \mathrm{L}$ of saline).

\section{Apparatus}

The behavioral procedures were performed in identical operant chambers $(24 \times 21 \times 30 \mathrm{~cm}$; Med Associates $)$ encased in sound attenuating cubicles. Each operant chamber was equipped with a food receptacle, a food dispenser that delivered one pellet (45-mg pellets; Bioserve) when activated, and a retractable lever on the left side of the food receptacle. Illumination was provided by a 24 $\mathrm{V} / 3 \mathrm{~W}$ house light mounted on the top-center of the wall opposite the food receptacle. The speaker that delivered the auditory conditioned stimuli was mounted on the wall opposite to the lever and the food receptacle. A computer system (MedPCSoftware; Med Associates) controlled the equipment and recorded the data.

\section{Behavioral procedure}

This experiment comprised three main stages: Pavlovian training, instrumental training, and transfer testing; in this latter stage, the effects of the Pavlovian stimuli on instrumental behavior were analyzed in extinction. Each session started with the illumination of the houselight and insertion of the lever where appropriate and ended with the retraction of the lever and turning-off of the houselight.

\section{Magazine training}

First, all subjects $(n=92)$ received one session of magazine training to habituate the animals to the operant chambers. During magazine training, food pellets (45-mg pellets; Bioserve) were delivered on a random time (RT) 30-sec schedule with no lever available.

\section{Pavlovian training}

Twelve sessions of Pavlovian training were given with the lever retracted. Two $80-\mathrm{dB}$ auditory stimuli $(3-\mathrm{kHz}$ tone and white noise) served as CS+ and CSo in a counterbalanced fashion. Each session contained six 2-min presentations of the CS+ followed by an interstimulus interval (ISI) of 2-4 min. Reward was delivered only during the presentation of the CS+ on a RT-30-sec schedule. For approximately half of the animals, the CS+ was the tone, and for the other half, the CS+ was the white noise. As a measure of Pavlovian conditioning, the total time the animal spent in the food receptacle during CS+ versus ISI was recorded via a photobeam in the food receptacle. 


\section{Instrumental training}

Following Pavlovian training, all animals received nine instrumental training sessions with the lever inserted. Responding on the lever was reinforced on a random interval (RI) schedule starting with RI-2-sec during the first session. For the next two sessions, the schedule was increased to RI-15-sec and RI-30-sec, and for the remaining six sessions to RI-60-sec. The total numbers of lever presses were recorded, and the session ended after $30 \mathrm{~min}$.

\section{Reminder and instrumental extinction}

Subsequent to instrumental training, one Pavlovian reminder was given to habituate unconditioned responses to the CSo. This session was similar to Pavlovian training sessions except that two additional, not reinforced, 2-min presentations of the alternative neutral stimuli (CSo) and additional ISI were given preceding the fifth and following the sixth presentation of the CS+. In addition, the animals received a single 30 -min instrumental extinction session with the lever available, but not reinforced.

\section{Transfer test}

The effect of Pavlovian stimuli on instrumental behavior was tested in extinction, while the lever was inserted into the operant chamber. Each stimulus (CS+ and CSo) was 2 min in duration and preceded by a 2 -min ISI period. The session always started with the presentation of the white noise, thus counterbalancing the order of presentation of CS+ and CSo within each group, and ended after four presentations of each stimulus type (CS+ and CSo). The total numbers of lever presses during the transfer test were recorded separately for CS+, CSo, and ISI. Subjects were assigned to five placement/drug/dose groups receiving either $0.5 \mu \mathrm{g}$ of SCH-23390 (NAC core, $n=11$; NAC shell, $n=7$ ), $0.75 \mu \mathrm{g}$ of SCH23390 (NAC core, $n=11$; NAC shell, $n=6$ ), $0.5 \mu \mathrm{g}$ of raclopride (NAC core, $n=11$; NAC shell, $n=7$ ), $1.0 \mu \mathrm{g}$ of raclopride (NAC core, $n=11$; NAC shell, $n=7$ ), or saline (NAC core, $n=13$; NAC shell, $n=8$ ) prior to the transfer test.

\section{Histology}

After completion of the experiment, all rats were euthanized with an overdose of sodium pentobarbital (150 mg/kg; Sigma-Aldrich) to control for correct cannulae placements. Brains were removed, fixed in $10 \%$ formalin for $24 \mathrm{~h}$, and stored in $30 \%$ glucose. Coronal brain sections $(40 \mu \mathrm{m})$ were collected, mounted on coated slides, and stained with cresyl violet. Cannulae placements were verified with reference to the atlas of Paxinos and Watson (1997).

\section{Statistical analysis}

Data from Pavlovian and instrumental training were subjected to an analysis of variances (ANOVA) for repeated measures with training day as factor. Pavlovian-instrumental transfer data were subjected to an ANOVA for repeated measures with treatment and CS identity as between-subject factor and stimulus as within-subject factor. Significant main effects and interactions were further investigated post hoc using the Tukey HSD test. Data were given as mean overall lever presses $( \pm$ SEM) during CS+, CSo, and ISI. For a detailed description of the data, the time course of stimulus effects in the transfer test, that is, lever presses during subsequent CS $+(4 \times)$, ISI $(8 \times)$, and CSo $(4 \times)$ presentations was also given. As the sequence of stimulus presentations in the transfer test was matched, i.e., one-half of rats received CS+/ISI/CSo/ISI . . . ISI/CSo, the other CSo/ISI/CS+/ISI . . . ISI/CS+, the data were collapsed such that the final ISI/CSo subsequence in one group and the initial CSo/ISI subsequence in the other were omitted. Increases of lever pressing during distinct $\mathrm{CS}+$ presentations relative to the preceding ISI were analyzed using planned contrasts. The level of statistical significance was $P<0.05$. All statistical analyses were carried out using STATISTICA Vers 7.1 (StatSoft).

\section{Acknowledgments}

This research was funded by a DFG grant Ha2340/6-3 (W.H.).

\section{References}

Anderson, S.M., Bari, A.A., and Pierce, R.C. 2003. Administration of the D1-like dopamine receptor antagonist SCH-23390 into the medial nucleus accumbens shell attenuates cocaine priming-induced reinstatement of drug-seeking behavior in rats. Psychopharmacology 168: $132-138$.

Baldo, B.A., Sadeghian, K., Basso, A.M., and Kelley, A.E. 2002. Effects of selective dopamine D1 or D2 receptor blockade within nucleus accumbens subregions on ingestive behavior and associated motor activity. Behav. Brain Res. 137: 165-177.

Bari, A.A. and Pierce, R.C. 2005. D1-like and D2 dopamine receptor antagonists administered into the shell subregion of the rat nucleus accumbens decrease cocaine, but not food, reinforcement. Neuroscience 135: 959-968.

Bassareo, V. and Di Chiara, G. 1997. Differential influence of associative and nonassociative learning mechanisms on the responsiveness of prefrontal and accumbal dopamine transmission to food stimuli in rats fed ad libitum. J. Neurosci. 17: 851-861.

Bassareo, V., De Luca, M.A., and Di Chiara, G. 2002. Differential expression of motivational stimulus properties by dopamine in nucleus accumbens shell versus core and prefrontal cortex. $J$. Neurosci. 22: 4709-4719.

Blundell, P., Hall, G., and Killcross, S. 2001. Lesions of the basolateral amygdala disrupt selective aspects of reinforcer representation in rats. J. Neurosci. 21: $9018-9026$

Caine, S.B., Heinrichs, S.C., Coffin, V.L., and Koob, G.F. 1995. Effects of the dopamine D-1 antagonist SCH 23390 microinjected into the accumbens, amygdala or striatum on cocaine self-administration in the rat. Brain Res. 692: 47-56.

Calaminus, C. and Hauber, W. 2006. Intact discrimination reversal learning but slowed responding to reward-predictive cues after dopamine D1 and D2 receptor blockade in the nucleus accumbens of rats. Psychopharmacology 191: 551-566.

Cardinal, R.N., Parkinson, J.A., Hall, J., and Everitt, B.J. 2002. Emotion and motivation: The role of the amygdala, ventral striatum, and prefrontal cortex. Neurosci. Biobehav. Rev. 26: 321-352.

Cardinal, R.N., Parkinson, J.A., Marbini, H.D., Toner, A.J., Bussey, T.J., Robbins, T.W., and Everitt, B.J. 2003. Role of the anterior cingulate cortex in the control over behavior by Pavlovian conditioned stimuli in rats. Behav. Neurosci. 117: 566-587.

Cheng, J.J., de Bruin, J.P., and Feenstra, M.G. 2003. Dopamine efflux in nucleus accumbens shell and core in response to appetitive classical conditioning. Eur. J. Neurosci. 18: 1306-1314.

Colwill, R.M. and Rescorla, R.A. 1988. Associations between the discrimintaive stimulus and the reinforcer in instrumental learning. J. Exp. Psychol. Anim. Behav. Process. 14: 155-164.

Corbit, L.H. and Balleine, B.W. 2005. Double dissociation of basolateral and central amygdala lesions on the general and outcome-specific forms of Pavlovian-instrumental transfer. J. Neurosci. 25: 962-970.

Corbit, L.H. and Janak, P.H. 2007. Inactivation of the lateral bit not medial dorsal striatum eliminates the ecitatory impact of Pavlovian stimuli on instrumental responding. J. Neurosci. 27: 13977-13981.

Corbit, L.H., Muir, J.L., and Balleine, B.W. 2001. The role of the nucleus accumbens in instrumental conditioning: Evidence of a functional dissociation between accumbens core and shell. J. Neurosci. 21: 3251-3260.

Corbit, L.H., Janak, P.H., and Balleine, B.W. 2007. General and outcome-specific forms of Pavlovian-instrumental transfer: The effect of shifts in motivational state and inactivation of the ventral tegmental area. Eur. J. Neurosci. 26: 3141-3149.

Datla, K.P., Ahier, R.G., Young, A.M., Gray, J.A., and Joseph, M.H. 2002. Conditioned appetitive stimulus increases extracellular dopamine in the nucleus accumbens of the rat. Eur. J. Neurosci. 16: 1987-1993.

de Borchgrave, R., Rawlins, J.N., Dickinson, A., and Balleine, B.W. 2002. Effects of cytotoxic nucleus accumbens lesions on instrumental conditioning in rats. Exp. Brain Res. 144: 50-68.

Di Chiara, G. and Bassareo, V. 2007. Reward system and addiction: What dopamine does and doesn't do. Curr. Opin. Pharmacol. 7: 69-76.

Dickinson, A. and Balleine, B. 2002. The role of learning in the operation of motivational systems. In Stevens's Handbook of experimental psychology (eds. H. Pashler and R. Gallistel), pp. 497-533. Wiley, New York.

Dickinson, A., Nicholas, D.J., and Adams, C.D. 1983. The effect of the instrumental training contingency on susceptibility to reinforcer devaluation. Q. J. Exp. Psychol. B 35: 35-51.

Dickinson, A., Smith, J., and Mirenowicz, J. 2000. Dissociation of Pavlovian and instrumental incentive learning under dopamine antagonists. Behav. Neurosci. 114: 468-483.

El-Amamy, H. and Holland, P.C. 2007. Dissociable effects of disconnecting amygdala central nucleus from the ventral tegmental 
area or substantia nigra on learned orienting and incentive motivation. Eur. J. Neurosci. 25: 1557-1567.

Estes, W.K. 1943. Discriminative conditioning. I. A discriminative property of conditioned anticipation. J. Exp. Psychol. 32: 150-155.

Hall, J., Parkinson, J.A., Connor, T.M., Dickinson, A., and Everitt, B.J. 2001. Involvement of the central nucleus of the amygdala and nucleus accumbens core in mediating Pavlovian influences on instrumental behaviour. Eur. J. Neurosci. 13: 1984-1992.

Holland, P.C. and Gallagher, M. 2003. Double dissociation of the effects of lesions of basolateral and central amygdala on conditioned stimulus-potentiated feeding and Pavlovian-instrumental transfer. Eur. J. Neurosci. 17: 1680-1694.

Ito, R., Dalley, J.W., Howes, S.R., Robbins, T.W., and Everitt, B.J. 2000. Dissociation in conditioned dopamine release in the nucleus accumbens core and shell in response to cocaine cues and during cocaine-seeking behavior in rats. J. Neurosci. 20: 7489-7495.

Kruse, J.M., Overmier, J.B., Konz, W.A., and Rokke, E. 1983. Pavlovian conditioned stimulus effects upon instumental choice behaviour are reinforcer specific. Learn. Motiv. 14: 165-181.

Lovibond, P.F. 1983. Facilitation of instrumental behavior by a Pavlovian appetitive conditioned stimulus. J. Exp. Psychol. Anim. Behav. Process. 9: 225-247.

Murschall, A. and Hauber, W. 2005. Effects of a systemic AMPA/KA and NMDA receptor blockade on Pavlovian-instrumental transfer. Psychopharmacology 182: 290-296.

Murschall, A. and Hauber, W. 2006. Inactivation of the ventral tegmental area abolished the general excitatory influence of Pavlovian cues on instrumental performance. Learn. Mem. 13: $123-126$

Nicola, S.M. 2007. The nucleus accumbens as part of a basal ganglia action selection circuit. Psychopharmacology 191: 521-550.

Ostlund, S.B. and Balleine, B.W. 2007. The contribution of orbitofrontal cortex to action selection. Ann. N. Y. Acad. Sci. 1121: 174-192.

Parkinson, J.A., Cardinal, R.N., and Everitt, B.J. 2000. Limbic cortical-ventral striatal systems underlying appetitive conditioning. Prog. Brain Res. 126: 263-285.
Paxinos, G. and Watson, C. 1997. The rat brain in stereotaxic coordinates. Academic Press, San Diego, New York.

Pecina, S., Schulkin, J., and Berridge, K.C. 2006. Nucleus accumbens corticotropin-releasing factor increases cue-triggered motivation for sucrose reward: Paradoxical positive incentive effects in stress? BMC Biol. 4: 8 . doi: 10.1186/1741-7007-4-8

Pellegrino, L.J., Pellegrino, A.S., and Cushmann, A.J. 1981. A stereotaxic atlas of the rat brain. Plenum Press, New York, London.

Rescorla, R.A. and Solomon, R.L. 1967. Two-process learning theory: Relationships between Pavlovian conditioning and instrumental learning. Psychol. Rev. 74: 151-182.

Trapold, M.A. and Overmier, J.B. 1972. The second learning process in instrumental learning. In Classical conditioning. II: Current research and theory (eds. A.A. Black and W.F. Prokasy), pp. 427-452. Appleton-Century-Crofts, New York.

Wilson, C., Nomikos, G.G., Collu, M., and Fibiger, H.C. 1995. Dopaminergic correlates of motivated behavior: Importance of drive. J. Neurosci. 15: 5169-5178.

Wyvell, C.L. and Berridge, K.C. 2000. Intra-accumbens amphetamine increases the conditioned incentive salience of sucrose reward: Enhancement of reward "Wanting" without enhanced "Liking" or response reinforcement. J. Neurosci. 20: 8122-8130.

Yun, I.A., Nicola, S.M., and Fields, H.L. 2004. Contrasting effects of dopamine and glutamate receptor antagonist injection in the nucleus accumbens suggest a neural mechanism underlying cue-evoked goal-directed behavior. Eur. J. Neurosci. 20: 249-263.

Zahm, D.S. 2006. The evolving theory of basal forebrain functional-anatomical 'macrosystems.' Neurosci. Biobehav. Rev. 30: $148-172$.

Zahm, D.S., Jensen, S.L., Williams, E.S., and Martin, J.R. 1999. Direct comparison of projections from the central amygdaloid region and nucleus accumbens shell. Eur. J. Neurosci. 11: 1119-1126.

Received February 25, 2008; accepted in revised form April 24, 2008. 


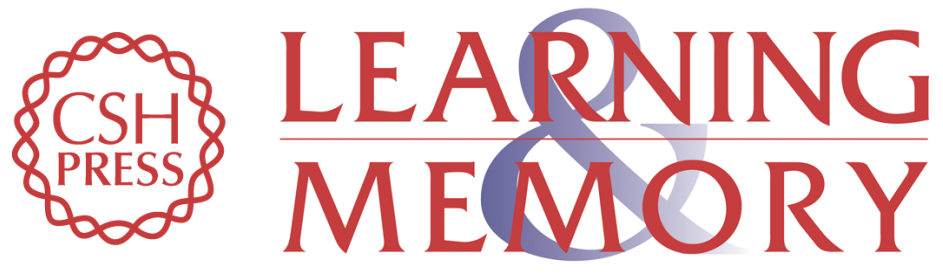

\section{Dopamine D1 and D2 receptors in the nucleus accumbens core and shell mediate Pavlovian-instrumental transfer}

Anja Lex and Wolfgang Hauber

Learn. Mem. 2008, 15:

Access the most recent version at doi:10.1101/lm.978708

References This article cites 40 articles, 10 of which can be accessed free at:

http://learnmem.cshlp.org/content/15/7/483.full.html\#ref-list-1

License

Email Alerting Receive free email alerts when new articles cite this article - sign up in the box at the Service top right corner of the article or click here. 